\title{
Social Inequalities in Health:Theory, Measurement and Evidence, With a Special Focus on Work and Employment
}

\author{
Johannes Siegrist ${ }^{1, *}$ \\ ${ }^{1}$ Faculty of Medicine, University of Duesseldorf, Germany \\ *Corresponding author: Johannes Siegrist, Senior Professorship, Faculty of Medicine, University of Duesseldorf, Germany. E-mail: johannes.siegrist@med.uni-duesseldorf.de
}

Received: 22 Sep 2016

Accepted: 21 Nov 2016

Ppub: 23 Dec 2016

\begin{abstract}
Background: Social inequalities in health between and within countries are considered a major public health challenge in economically developed and developing countries. Population groups living in socially disadvantaged conditions experience poorer health and shorter life expectancy than those who are more privileged. To a high extent these inequalities are subject to modification by policy programs strengthening prevention. Yet, to this end solid research evidence on the description and explanation of health inequalities is needed.

Methods: This lecture provides some basic knowledge to this end. First, major concepts and methods of assessing social inequalities within different types of societies are outlined. Individual-level measures of social stratification (education, occupational status, income) are of primary importance. Second, selected main findings of epidemiological research on social inequalities in morbidity and mortality are demonstrated. Following this, three promising areas of explaining health inequalities are presented. They concern (a) adverse social circumstances during pregnancy and in early childhood, (b) health-adverse behaviors acquainted during adolescence and early adulthood (especially unhealthy diet, physical inactivity, smoking), and (c) material and psychosocial adversity in adult life, in particular with regard to work and employment.

Results: Own research focusing on health-adverse working conditions in different parts of the world has demonstrated substantial social inequalities in access to and quality of work. People with low socioeconomic status are more often exposed to material and psychosocial adversity at work, including low control and an imbalance between high effort spent and low reward received in turn. These inequalities contribute to a higher burden of work-related diseases (in particular cardiovascular disease and depression).

Conclusions: Finally, the lecture emphasizes the importance of convincing research evidence on social inequalities in health as this evidence offers definite entry points for preventive policy programs. Once developed and implemented such programs can contribute to the reduction of avoidable inequalities in health.
\end{abstract}

Keywords: Health Inequalities; Social Determinants; Public Health; Working Conditions and Health; Prevention Programs 